\title{
Focal Point Theory Models for Dissecting Dynamic Duality Problems of Microbial Infections
}

\author{
S.-H. Huang, ${ }^{1}$ W. Zhou, ${ }^{2}$ and A. Jong ${ }^{1}$ \\ ${ }^{1}$ Childrens Hospital Los Angeles, University of Southern California, Los Angeles, CA 90027, USA \\ ${ }^{2}$ HRL Laboratories, LLC., Malibu, CA 90265, USA
}

Correspondence should be addressed to S.-H. Huang, shhuang@hsc.usc.edu

Received 31 August 2007; Accepted 19 December 2007

Recommended by Daniel Howard

Extending along the dynamic continuum from conflict to cooperation, microbial infections always involve symbiosis (Sym) and pathogenesis (Pat). There exists a dynamic Sym-Pat duality (DSPD) in microbial infection that is the most fundamental problem in infectomics. DSPD is encoded by the genomes of both the microbes and their hosts. Three focal point (FP) theory-based game models (pure cooperative, dilemma, and pure conflict) are proposed for resolving those problems. Our health is associated with the dynamic interactions of three microbial communities (nonpathogenic microbiota (NP) (Cooperation), conditional pathogens (CP) (Dilemma), and unconditional pathogens (UP) (Conflict)) with the hosts at different health statuses. Sym and Pat can be quantitated by measuring symbiotic index (SI), which is quantitative fitness for the symbiotic partnership, and pathogenic index (PI), which is quantitative damage to the symbiotic partnership, respectively. Symbiotic point (SP), which bears analogy to FP, is a function of SI and PI. SP-converting and specific pathogen-targeting strategies can be used for the rational control of microbial infections.

Copyright (C) 2008 S.-H. Huang et al. This is an open access article distributed under the Creative Commons Attribution License, which permits unrestricted use, distribution, and reproduction in any medium, provided the original work is properly cited.

\section{INTRODUCTION}

Infectious diseases caused by bacterial, viral, fungal, or parasitic pathogens continue to be the leading cause of morbidity and mortality worldwide despite the availability of effective antimicrobial agents and vaccines over the last fifty years or more [1]. The continual emergence of previously undescribed new pathogens, reemergence of old pathogens, and the rising crisis of antibiotics resistance will certainly heighten the global impact of microbial infections in the 21st century. These problems are mainly due to inadequate knowledge of the dynamic duality relationships between symbiosis (Sym) and pathogenesis (Pat) in microbial infections [2]. The term symbiosis, which may have many variations on its definition, in this paper refers to living together through a close and prolonged association between two or more organisms of different species [3, 4]. Duality is defined as different ways of looking at the same thing [5]. There are two major limitations inherent in the conventional theories of microbial infection. On the one hand, in the past century, biology and medicine including infectious diseases have been dominated by the reductionistic ap- proaches. Focusing research on individual virulence genes and the important pathogens has been the traditional approach to human infectious diseases. On the other hand, as Joshua Lederberg pointed out $[6,7]$, medical science is imbued with the Manichaean view of the microbe-human host relationship: "we good; they evil." Almost all broad-spectrum antimicrobial agents, which are in the best interest of pharmaceutical industries, kill both the good microbes as well as the bad germs. Even though narrow-spectrum antiinfective agents are not "narrow" for pathogens, they also target both the good and bad microorganisms with a limited range of species.

Animals and plants are continually infected by an extensive diversity of symbiotic or invading organisms including bacteria, virus, fungus, or parasites. Infection of bacteria by phages started long before the emergence of animals and plants [8]. Microbial infection is an evolutionary paradigm which is associated with coevolution between hosts and microbes $[6,7,9]$. This coevolution can be defined as the process of reciprocal and dynamic genetic changes in two or more species [2]. The conventional wisdom in medicine holds that microbial infection is a pathogenic process in 


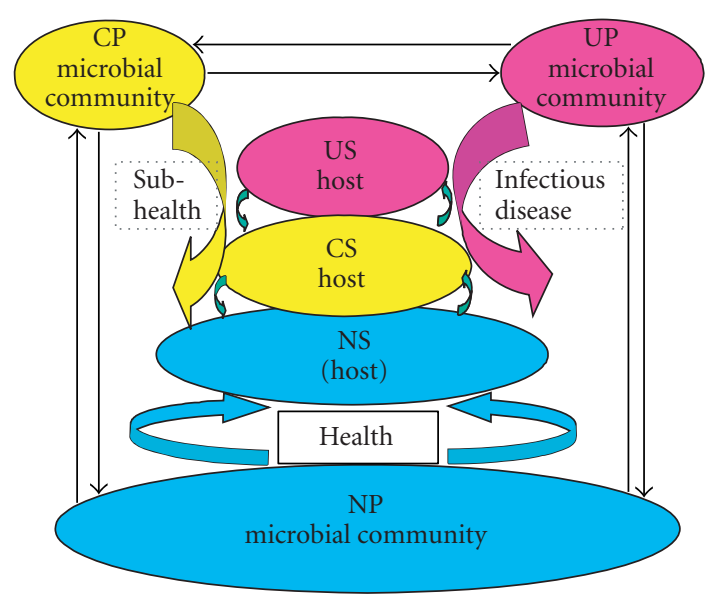

FIGURE 1: Schematic representation of interactions of three microbial communities (nonpathogenic (NP), conditional pathogenic (CP), and unconditional pathogenic (UP)) with the hosts at three different health statuses (nonsusceptibility (NS), conditional susceptibility (CS), and unconditional susceptibility (US)).

which a pathogen enters, establishes itself, and multiplies in the host [10]. The emphasis is on the antagonism or conflict, not the mutualism. This represents "zero-sum thinking"the belief that if one player gains, other player must inevitably lose. Methods and concepts of the zero-sum game theory have proved successful in studying the strategy of pure conflict. The most challenging issue in infectious diseases is how to dissect the dynamic Sym-Pat duality (DSPD) in microbial infections using infectomics and mathematics such as focal point- (FP-) based game theory. Game theory, defined in the broadest sense, is the study of the strategies of conflict, cooperation, and mixed situations in which both coexist. Generally, there are multiple equilibria in a game. Thomas Schelling's concept of focal point, which is an equilibrium usually standing out from the others, addressed the crucial question of how to interpret the multiplicity of equilibria $[11,12]$. Focal point, the principal component of Schelling's game theory, is a convergence point of expectations about actions in a game. This article attempts to enlarge the scope and application of focal point game theory in microbial infections, extending from the zero-sum games to the nonzerosum games.

\section{DEFINITIONS AND METHODS}

\subsection{Three-community principle of microbial infections}

Our health is associated with the dynamic interactions of three microbial communities [2] (nonpathogenic microbiota (NP), conditional pathogens (CP), and unconditional pathogens (UP)) with the hosts at three different health statuses (nonsusceptibility (NS), conditional susceptibility (CS), and unconditional susceptibility (US)) (see Figure 1). $\mathrm{NP}$ is the major microbial community which forms a healthy symbiotic "superorganism" with the hosts. The ecology and evolution of NP-NS interaction are essential and fundamental for health. From birth to death, we share a benign coexistence with a vast, complex, and dynamic consortium of microbes. Most of our microbial commensals reside in our gastrointestinal (GI) track packed with up to 100 trillion $\left(10^{14}\right)$ microbes $[1,13]$. The GI tract harbors a rich microbiota of $>600$ different bacterial species. Some of these microorganisms have important health functions. These include stimulating the immune system, protecting the host from microbial invasion, and aiding digestion. The gut microbiota, which is essential for human homeostasis, is established rapidly after birth and remains relatively stable throughout the life [1]. The GI mucosa provides a protective interface between the internal environment and the constant external challenge from food-derived antigens and microbes. CP and UP are minor microbial communities that mainly contribute to the pathogenesis of microbial diseases. The distinction between the commensal and the pathogen in the $\mathrm{CP}$ community can be blurred because they may cause diseases under certain sub-health conditions of the hosts, or in immunocompromised hosts. For example, pneumococcus, meningococcus, and Haemophilus bacteria regularly exist as part of the normal microbiota of the host respiratory track and are mostly carried asymptomatically despite the fact that they can cause well-defined diseases [14, 15]. Microbes in the CP community dynamically evolve in two opposite directions, which are toward either the NP (more cooperative or mutualistic) or UP (more pathogenic) microbial community. Microbes with high pathogenicity belong to the UP microbial community. The three microbial communities and three statuses of the hosts are subjected to dynamic reciprocal changes driven by transfer of genetic materials.

\subsection{Dynamic duality relationships between Sym and Pat in microbial infections}

Extending along the dynamic continuum from conflict to cooperation, microbial infections always involve symbiosis and pathogenesis, which are two fundamental components of the host-microbe interactions (see Figure 2). There exists a dynamic Sym-Pat duality in microbial infection, which is the most fundamental issue of infectomics [2]. DSPD is reflected in the genotypic and phenotypic infectomes, which are encoded by the genomes of both the microbes and their hosts [2]. The opposition and unity of Sym and Pat are indispensable, and the academic viewpoint that the unity of opposites of Sym and Pat gives impetus to the development of microbial infection is considered as the core idea and radical principle of the duality representations of microbial infections. In certain circumstances and at a certain stage of the development of microbial infection, each of the two aspects of Sym and Pat will transform from antagonism into mutualism or from mutualism into antagonism. Sym and Pat can be quantitated by measuring symbiotic index (SI), which is quantitative fitness for the symbiotic partnership, and pathogenic index (PI), which is quantitative damage to the symbiotic partnership, respectively. The most crucial studies are to identify 


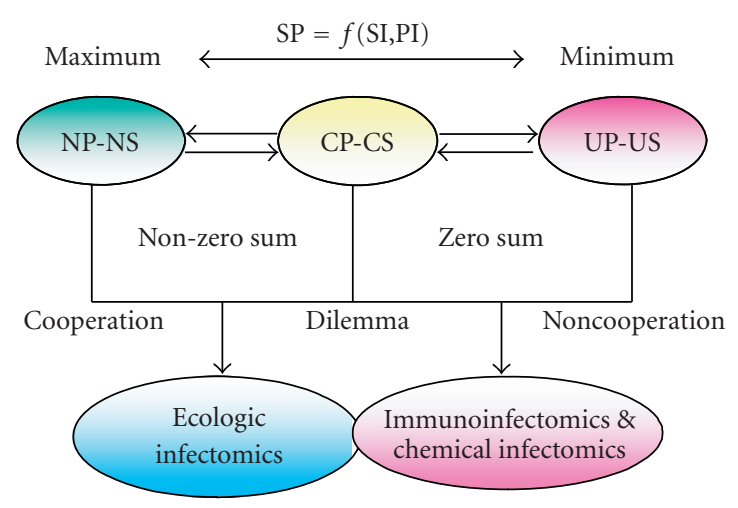

FIgURE 2: A continuum model of host-microbe interactions coupling with infectomic approaches to dissect the problems in microbial infections.

infectomic signatures specific for SI and PI. The set of symbiotic or pathogenic parameters is defined as a function $\operatorname{SI}(x)$ or $\mathrm{PI}\left(x^{*}\right) . \mathrm{SI}(x)$ and $\mathrm{PI}\left(x^{*}\right)$ are continuous functions ranging from 0 to 1 to admit different degrees of Sym and Pat, respectively. $\operatorname{SI}(x)=0$ and $\operatorname{PI}\left(x^{*}\right)=0$ indicate that $x$ and $x^{*}$ are perceived to be zero-symbiotic and zero-pathogenic, respectively. $\operatorname{SI}(x)=1$ and $\operatorname{PI}\left(x^{*}\right)=1$ indicate that $x$ and $x^{*}$ are perceived to be completely symbiotic and completely pathogenic, respectively. Intermediate values of $\operatorname{SI}(x)$ and $\mathrm{PI}\left(x^{*}\right)$ indicate that $x$ and $x^{*}$ are perceived to be partially symbiotic and partially pathogenic, respectively. Symbiotic points are used to determine the dynamic duality between Sym and Pat. SI and PI are interdependent parameters. The symbiotic point (SP) is a function of SI and PI:

$$
\mathrm{SP}=f(\mathrm{SI}, \mathrm{PI})
$$

The focus of the dynamic duality research is to examine the ability of SP to transform situations of potential conflict (UPUS and CP-CS) into situations of cooperation (NP-NS). SP bears analogy to Schelling's focal point, which is any feature of such a game that provides a focus of convergence [16]. In the games with multiple Nash equilibria, one equilibrium usually stands out from the others (salient). Such an equilibrium is a focal point which can be easily recognized by all the players [12]. Thomas Schelling's Strategy of Conflict (1960) has been recognized as one of the most important works of game theory $[11,17]$. There is no doubt that focal points play a central role in Schelling's game theory. Schelling has made a significant contribution to a reorientation of game theory. Understanding focal points is not only a key to improving game theory but also a key to dissecting SPs.

\subsection{Game theoretical models (GTMs) of microbial infections}

In this paper, three types of GTMs are proposed for studies on NP-NS interactions (cooperative game), UP-US interactions (noncooperative games), and CP-CS interactions (dilemma or bargaining game). First, the NS-NS interactions are dissected with pure cooperative games in which each player chooses the strategy corresponding with the focal point in the expectation that the others will do the same. The significance of focal points can be shown most clearly in the pure cooperative games. As there is no conflict of interests in these games, all the players merely want to cooperate and they do not choose the alternative ways. Analysis of the cooperative game issues is to focus on coalition formation and distribution of the gains through cooperation. The SP in the NP-NS games tends to be maximal (see Figure 2). Secondly, noncooperative GTMs are used for analysis of the UP-US interactions. In contrast to cooperative games which focus on collective rationality and common interest, noncooperative games emphasize individual rationality and individual optimal strategy. The SP in the UP-US games tends to be minimal (see Figure 2). In games of pure conflict, defection is the equilibrium strategy and the total benefit to all players in the game, for every combination of strategies, always adds to zero (zero-sum). In the antagonistic UP-US interaction model, the surviving strategies of the UP community conflict with that of the US host. The UP evolves to exploit the host as much as possible, and the host adapts to exclude or limit the damage caused by the UP. Thirdly, we consider the strategic use of focal point theory in mixed situations to analyze the CP-CS interactions in which there is both conflict and mutual dependence. The most well-known example is the Prisoner's Dilemma game (a two-player game) in which each player chooses between a cooperating and defecting strategy. In this game, each player receives a higher playoff by defecting than by cooperating. However, a higher playoff is received if both cooperate than both defect. The two-player game can be extended to the N-player Prisoner's Dilemma game with arbitrary numbers of players.

\section{RESULTS}

\subsection{Three communities in Escherichia coli species}

E. coli is one of the best understood and most thoroughly studied organisms and is advantageous as a model microorganism for the current studies. This bacterium is genotypically and phenotypically a highly diverse species, which is present in the three microbial communities (see Table 1). Most E. coli strains are commensals of higher vertebrates belonging to NP, but some are pathogenic (CP and UP). Uropathogenic E. coli (UPEC) in the CP group are the most common cause of community-acquired urinary tract infection (UTI). UPEC are responsible for about $80 \%$ of the estimated 150 million UTIs diagnosed annually [18]. E. coli $\mathrm{O} 157$, which belong to the UP group, is a major food pathogen. Shigella species, the cause of dysentery, are now known to be multiple distinct lineages of $E$. coli. Genomes of those E. coli strains have been sequenced (see Table 1). Recently, "better" E. coli strains (MDS41, 42, 43) have been engineered in which about $15 \%$ of the genome has been removed with the use of synthetic biology [19]. Coliphage, a virus which infects $E$. coli, is a major contributor responsible for diversification of $E$. coli [20,21]. From a populationdynamic view, the interactions between coliphage and E. coli are analogous to those of a predator and a prey. 
TABLe 1: E. coli in the three microbial communities.

\begin{tabular}{|c|c|c|c|c|c|}
\hline & \multirow{2}{*}{ E. coli strains } & \multirow{2}{*}{ characteristics } & \multirow{2}{*}{ Genome (Mb) } & \multicolumn{2}{|c|}{ Putative } \\
\hline & & & & SI & PI \\
\hline \multirow{4}{*}{ NP } & MG1655 (K12) & Commensal & 4.6 & $>0.75$ & $<0.25$ \\
\hline & Nissle 1917 & Probiotics & 5.1 & $>0.75$ & $<0.25$ \\
\hline & A0 34/86 & Probiotics & 4.8 & $>0.75$ & $<0.25$ \\
\hline & MDS41, 42, 43 & K12 strains & 3.9 & $>0.75$ & $<0.25$ \\
\hline \multirow{2}{*}{$\mathrm{CP}$} & RS218 & Low pathogenicity & 5.1 & $0.5 \pm 0.25$ & $0.5 \pm 0.25$ \\
\hline & CFT073 & Uropathogenicity & 5.2 & $0.5 \pm 0.25$ & $0.5 \pm 0.25$ \\
\hline \multirow{3}{*}{ UP } & O157 RIMD & High pathogenicity & 5.5 & $<0.25$ & $>0.75$ \\
\hline & O157 EDL & High pathogenicity & 5.5 & $<0.25$ & $>0.75$ \\
\hline & Shigella Sd197 & High pathogenicity & 4.4 & $<0.25$ & $>0.75$ \\
\hline
\end{tabular}

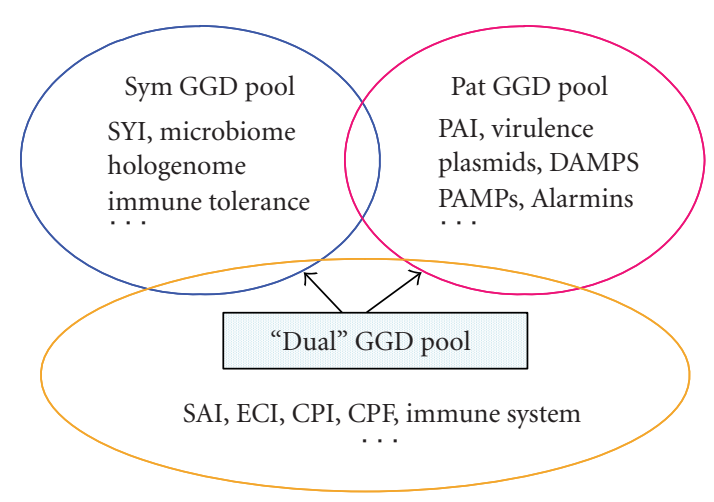

FIgUre 3: Gene pools contributing to the Sym-Pat duality. CPF: CP factors; CPI: CP islands; ECI: ecological islands; HPI: high pathogenicity islands; PAI: pathogenicity islands; SYI: symbiosis island.

\subsection{Sym-Pat duality is encoded by the genomes of both microbes and their hosts}

The development of microbial infections depends on the dynamic Sym-Pat duality, which is governed by the genomes of both the microbes and their hosts [2]. Molecular evolution of genetic structures for the Sym-Pat duality is influenced by both biotic and abiotic environmental factors in the ecosystems. There are three types of genetic/genomic determinants (GGDs) that may contribute to the Sym-Pat duality of microbial infections under specific environmental conditions (see Figure 3). The first type is the Sym GGD pool, which contributes to symbiosis. The Sym GGDs from the microbial partner include symbiosis-related genomic islands (SYI), plasmids, transposons, and microbiome, which is a collective genome of microbiota $[2,22,23]$. The gene pool contributing to microbial tolerance belongs to the host Sym GGDs [24]. The symbiotic homeostasis of the superorganism formed by the microbiota and its host is governed by hologenome, a complex of the host genome and microbiome $[22,25]$. The second pool of GGDs contributes to the pathogenesis of microbial infections. These include pathogenicity islands (PAI), virulence plasmids, pathogen-associated molecular patterns (PAMPs), and endogenous alarmins [26].
PAMPs are a diverse group of microbial molecules, which are recognized by the host innate and adaptive immune system, primarily through toll-like receptors (TLRs) [26]. Alarmins are endogenous molecules within the host that signal tissue and cell damage. Effector cells of the innate and adaptive immunity can release alarmins when they are activated by PAMPs. Endogenous alarmins and exogenous PAMPs elicit similar responses by conveying a similar message. Therefore, they constitute a larger family of damage-associated molecular patterns (DAMPs). The third type of GGD pool has dual functions that depend on external and internal environments. These include ecological islands (ECI), certain GGDs from conditional pathogens (such as CP factors (CPFs) and CP islands (CPIs)), and the host GGDs with dual effects on microbial infections. The dual GGDs contribute to the Sym and Pat duality in specific ecological niches and within particular organisms. The same GGD may act as an SYI when the microbial recipient establishes a symbiotic relationship with its host, but becomes a PAI when it is adapting the pathogenic niche. A comparative infectomic study suggests that GimA, a $20-\mathrm{kb}$ genomic island, is a typical CPI [27]. The dual biological functions of GimA depend on the genomic environments in E. coli strains. GimA present in meningitic E. coli $\mathrm{K} 1$ genome $(\mathrm{O} 18: \mathrm{K} 1: \mathrm{H} 7)$ is essential for bacterial crossing the blood-brain barrier to cause meningitis [28]. In contrast, GimA is required for the probiotic function of E. coli K24 strain A0 34/86 (O83:K24:H31), which has been safely and effectively used in Czech pediatric clinics since 1967 [29]. The dual Sym-Pat properties of microbial determinants were also observed in photorhabdus, which is a genus of Gram-negative bacteria mutualistically associated with entomophagous nematodes of the family heterorhabditiae [30]. A hexA homologous gene from photorhabdus is able to regulate both symbiosis and pathogenesis [30]. Some microbes exhibit dual behavior as symbionts and pathogens in a manner dependent on the hosts. Sooty mangabey (SM) monkeys infected with simian immunodeficiency virus (SIV) do not develop acquired immunodeficiency syndrome (AIDS) [31]. In contrast, SIV infection of non-natural host monkeys, such as rhesus macaques (RMs), causes AIDS that closely resembles the human disease [31]. Similarly, polydnaviridae, a family of double-stranded DNA viruses, have evolved complex life cycles in which they interact as symbionts with one host 
and as pathogens with another [32]. All multicellular organisms, including human and flies, have evolved the conservative innate immune system as a double-edged sword [33]. It enables the host not only to combat pathogens but also to develop microbial tolerance to cohabit nonpathogenic microbiota by maintaining the homeostatic balance between the host and microorganisms [24, 33]. TLRs play central roles in the activating process of the innate immune system with dual functions. They have recently been shown to be involved in modulating intestinal homeostasis by recognizing commensal bacteria. They also sense extracellular PAMPs by triggering signaling, which results in the activation of proinflammatory (PI) pathways [24]. PI ligands of TLRs may be important for the activation and expansion of natural $\mathrm{T}$ regulatory cells (NatTReg), which control both deleterious and protective immune responses upon microbial infections [34]. Both innate TLRs and specific T cell receptors (TCR) contribute to the dual functions of NatTReg [34]. The full range of the dual GGDs in the immune system is unknown so far. However, it can be expected that the molecular evolution of the dual GGDs during the host-microbe coevolution will certainly lead to dynamic changes in the Sym-Pat duality.

\subsection{Duality relationship between Sym and Pat}

Sym and Pat, the fundamental components of microbial infection, can be defined as a function $\operatorname{SI}(x)$ or $\operatorname{PI}\left(x^{*}\right)$. $\operatorname{SI}(x)$ and $\operatorname{PI}\left(x^{*}\right)$ are continuous functions ranging from 0 to 1 to admit different degrees of Sym and Pat, respectively (see Figure 4). $\operatorname{SI}(x)=0$ and $\operatorname{PI}\left(x^{*}\right)=0$ indicate that $x$ and $x^{*}$ are perceived to be zero-symbiotic and zero-pathogenic, respectively. $\operatorname{SI}(x)=1$ and $\operatorname{PI}\left(x^{*}\right)=1$ indicate that $x$ and $x^{*}$ are perceived to be completely symbiotic and completely pathogenic, respectively. If SI is close to or equal to $1, \mathrm{mi}-$ crobial infection is a physiological process. It is now well accepted that mitochondria were derived from an endosymbiotic relationship with internalized proteobacteria, via a progressive transfer of genetic material [35]. This long symbiotic relationship reaches the maximum Sym value. The symbiotic nitrogen fixation process for converting atmospheric dinitrogen $\left(\mathrm{N}_{2}\right)$ to ammonia $\left(\mathrm{NH}_{3}\right)$ is essentially dependent on two partners: the host legume plant and bacteria belonging to the family Rhizobiaceae [36]. This type of microbial infection is more typically associated with a physiological process.

If the PI value approaches or reaches the maximum limit, microbial infection is more completely associated with a pathogenic process. Most of serious infectious diseases fall into this category. Intermediate values of $\operatorname{SI}(x)$ and $\operatorname{PI}\left(x^{*}\right)$ indicate that $x$ and $x^{*}$ are perceived to be partially symbiotic and partially pathogenic, respectively. Microbial infections induced by conditional pathogens represent a competitive relationship (see II or III in Figure 4). The hosts have large influences on SI and PI. For example, polydnaviruses have evolved complex life cycles in which they are able to adapt to a mutualistic partnership with one host and become pathogens with another. Their genomes reflect the dual roles as mutualists and pathogens [32].

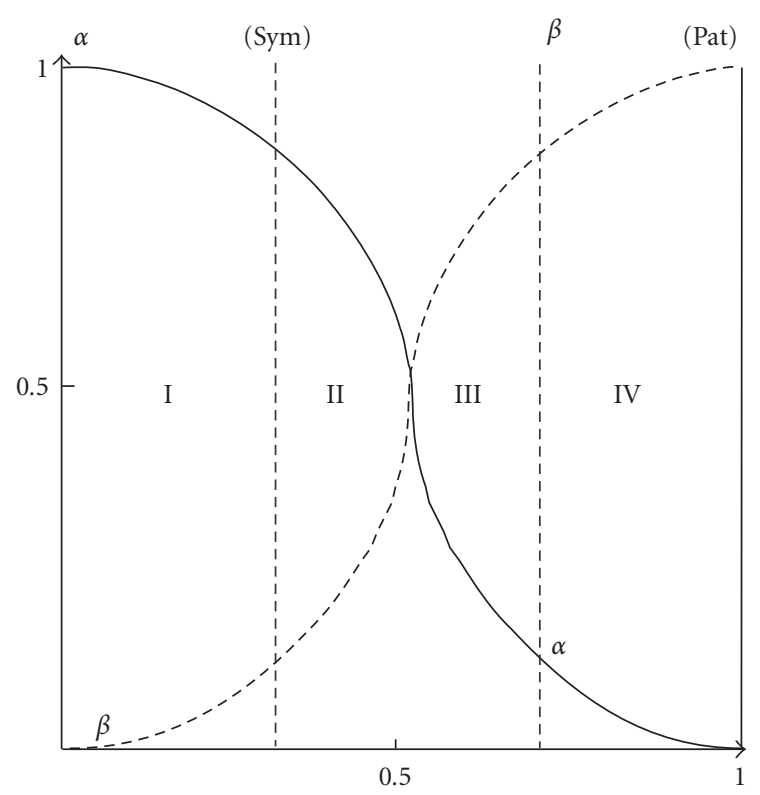

FIgUre 4: The duality relationship between Sym $\left(\alpha_{i}\right)$ and Pat $\left(\beta_{i}\right)$. A cooperative relationship (NP-NS: health and mutualism) (I) occurs between the host and the NP microbial community. A competitive relationship (CP-CS) (II and III) exists between the host and the CP microbial community. There are two types of competitions: better (II) and worse (III). An antagonistic relationship (UP-US) (IV) occurs between the host and the UP microbial community.

\subsection{Outcomes of three types of games in microbial infections}

The outcome of a game is not only determined by one individual's choices, but also depends on the strategies used by all the others. The dynamic Sym-Pat duality influenced by both microbes and their hosts is the key factor that determines the outcome of a game associated with microbial infection. One of the most fundamental issues in game theoretical solution concepts is that strategies used by individual players are based on the differences in payoff perceived by them [37]. This issue can be solved by Schelling's focal point theory. FPs constitute shared expectations that coordinate the activities of diverse players collectively or independently seeking their goals [38]. By harmonizing anticipated behaviors or responses despite the presence of imperfect information, individuals are able to coordinate their activities towards their ends. In 1960, Schelling classified games into three major categories: pure cooperative game on one side, pure conflict games on the other, and combinations of partial cooperation/partial conflict games in between [11]. Recently, a similar classification was designated in Gao's book, "Principles of Systemics" [39]. The pure cooperative game models are used for dissecting NP-NS interaction problems. The payoff matrix for a pure NP-NS problem would resemble something like that in Figure 5(a). In contrast, the pure UP-US interaction, a situation of pure antagonism, is characterized by completely opposing interests, where the pathogenesis of microbial infection is the most predominant event. The payoff matrix for the pure UP-US game would look something like that 


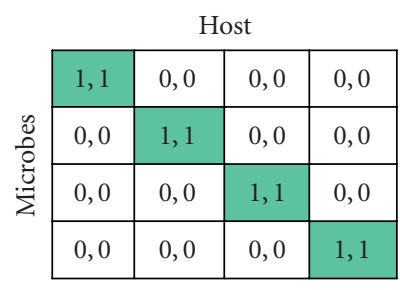

(a)

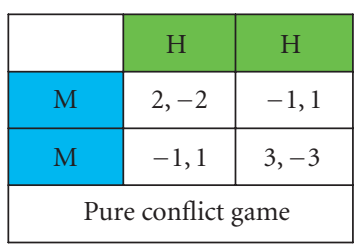

(b)

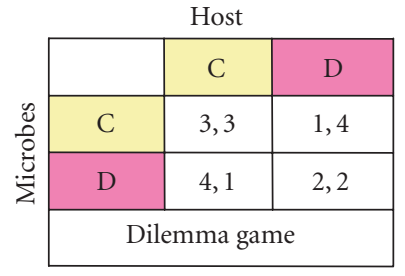

(c)

Figure 5: (a) Pure cooperative game. (b) Pure conflict game. H: host; M: microbes. (c) Dilemma game. The number in the left of each pair indicates the payoff for microbes, and the right, the host. Higher numbers represent greater payoff for the individual. Two strategies (Cooperation (C) and Defection (D)) are used.

in Figure 5(b). The state of microbial infection has conventionally been characterized as lying on the extreme conflict end. This depiction comes from the Manichaean view of the microbe-human host relationship. This situation is depicted in Figure $5(\mathrm{~b})$ as a two-player game. In the pure conflict game, the relationships between the microbes and their hosts end up in a "war of all against all" in which the payoffs of the outcomes add to zero (zero-sum). The three microbial community principle and the dynamic Sym-Pat duality concept would help establish a holistic view of microbial infections. The CP-CS interaction is a situation where cooperation and conflict coexist. As illustrated in Figure 5(c), the payoffs for the CP-CS games would lie midway between the pure cooperative and pure conflict games. The CP-CS problems are microbial dilemmas, in which there is a mixture of mutual dependence and conflict of partnerships and competition. The underlying idea arises naturally from the well-known games for the social dilemmas and the Prisoner's Dilemma (PD), in which each player chooses between a cooperating and a defecting strategy [40]. As shown in Figure 5(c), each player receives a higher playoff by defecting than by cooperating, no matter what the other player chooses. However, they receive a higher playoff if both cooperate than both defect. The CPCS interactions can coevolve toward two different directions, increasing (more cooperation) or decreasing (more antagonism) the SP (see Figure 2).

\section{DISCUSSION}

In this paper, focal point theory-based game models are proposed for analysis of the dynamic Sym-Pat duality in microbial infections. DSPD is the most fundamental problem in infectomics, which is the integration of omics and mathematical/computational approaches. There are three types of infectomic approaches that can be used for the control of microbial infections: ecological infectomics, immunoinfectomics, and chemoinfectomics [2]. Ecological infectomics will explore symbiotic solutions to microbial infections. Developing novel immunological intervention strategies for the prevention and treatment of microbial infections using infectomic signatures and immunomic approaches falls within the field of immunoinfectomics. Chemoinfectomics represents the most powerful approach to the development of a new generation of drugs for antimicrobial chemotherapy.

\subsection{Symbiosis point converting (SPC): ecological infectomics-based approaches for rational control of microbial infections}

As microbial infection is an ecological and evolutionary paradigm which is associated with coevolution between hosts and microbes (such as human host and microorganisms, phages, and bacteria) in dynamic ecosystems, two ecological infectomics-based SPC approaches (increasing and decreasing SP) can be used for rational control of infectious diseases [2]. The focus in SP increasing approaches is how to transform situations of potential conflict (pathogenesis) into cooperation (symbiosis) by dissecting the dynamic duality relationships between Sym and Pat in microbial infections and developing symbiotic agents (symbiotics) that favor a healthy symbiosis [2]. Symbiotics are defined as products that are beneficial to symbiotic ecology of the superorganisms consisting of microbes and their human hosts. These include microbial (e.g., probiotic bacteria) and nonmicrobial agents (e.g., prebiotics) [2]. The introduction of beneficial symbiotics with higher SP in our body should be a very attractive rationale for modulating the microbiota, improving the symbiotic homeostasis of the superorganism, and providing a microbial stimulus to the host immune system against pathogens. The use of probiotics has been suggested as a promising approach for combating infectious diseases, and delivering drugs and vaccines [2]. Decreasing SP is another rational strategy for control of microbial infections. As phages, which specifically kill bacteria, play an important role in the ecology, evolution, and virulence of a number of pathogens, there is a rational use of phages for treatment and prevention of bacterial infections. The use of phages to treat bacterial infections has a long history dating back to mid 1910's [2]. Due to the availability of effective broadspectrum antibiotics in the early 1940's, phage therapy was discarded in Western medicine at that time. The rising crisis of antibiotic resistance has recently increased great interest in phages and their use as natural antimicrobial agents to fight microbial infections [2]. Compared with commonly used antibiotics, a great advantage of phages is their narrow host range. Recent studies have shown that coinfection with GB virus $\mathrm{C}(\mathrm{GBV}-\mathrm{C})$ is associated with a decreased mortality in HIV-infected patients [41]. Therefore, reducing SP between microbial agents (such as phages and GBV-C) and targeted 
pathogens is another excellent ecological approach for the development of novel antimicrobial agents.

\subsection{Specific pathogen-targeting (SPT): immunoinfectomics- and chemoinfectomics-based approaches for prevention and treatment of infectious diseases}

In contrast to the ecological infectomics-based SPC approaches that focus on the symbiotic relationships (such as NP-NS and CP-CS interactions) between the hosts and microbial communities, immunoinfectomics- and chemoinfectomics-based SPT approaches emphasize the use of antagonistic relationships (such as UP-US interactions) between the hosts and microorganisms. It is important to point out that the SPT approaches are intrinsically different from the conventional pathogen-targeting antimicrobial agents, which kill both pathogens and nonpathogens [2]. The availability of the genomic information from both microbes and their hosts has resulted in exciting new progress in the field of immunoinfectomics. Nanobody (the smallest fragment of naturally occurring single-domain antibody)-based technologies and immune epitope mapping have emerged as the very powerful tools for the discovery and development of novel antimicrobial agents [2]. Recently, a nanobodyconjugated human trypanolytic factor has been successfully used for an experimental therapy of African trypanosomiasis [42]. Concurrent advances in both high-throughput chemistry and infectomics have given rise to the field of chemoinfectomics for elucidating and validating drug targets, and generating novel therapeutics. Chemoinfectomics refer to the use of small synthetic molecules that are highly specific for defined infectomic targets, for biological function analysis and to discover new drug leads. The progress towards understanding the dynamic Sym-Pat duality in microbial infections using focal point theory-based game models will greatly facilitate the use of ecological infectomics, immunoinfectomics, and chemoinfectomics for the rational control of infectious diseases.

\section{REFERENCES}

[1] S.-H. Huang, T. Triche, and A. Jong, "Infectomics: genomics and proteomics of microbial infections," Functional \& Integrative Genomics, vol. 1, no. 6, pp. 331-344, 2002.

[2] S.-H. Huang, X. Wang, and A. Jong, "The evolving role of infectomics in drug discovery," Expert Opinion on Drug Discovery, vol. 2, no. 7, pp. 961-975, 2007.

[3] M. J. Roossinck, "Symbiosis versus competition in plant virus evolution," Nature Reviews Microbiology, vol. 3, no. 12, pp. 917-924, 2005.

[4] G. G. Dimijian, "Evolving together: the biology of symbiosis," Baylor University Medical Center Proceedings, vol. 13, no. 3, pp. 217-226, 2000.

[5] L. Smolin, Three Roads to Quantum Gravity, Basic Books, New York, NY, USA, 2001.

[6] J. Lederberg, "Infectious history," Science, vol. 288, no. 5464, pp. 287-293, 2000.
[7] J. Lederberg, “The future of infectious diseases," Journal of Urban Health, vol. 75, no. 3, pp. 463-470, 1998.

[8] H. Brüssow, C. Canchaya, and W.-D. Hardt, "Phages and the evolution of bacterial pathogens: from genomic rearrangements to lysogenic conversion," Microbiology and Molecular Biology Reviews, vol. 68, no. 3, pp. 560-602, 2004.

[9] M. E. J. Woolhouse, J. P. Webster, E. Domingo, B. Charlesworth, and B. R. Levin, "Biological and biomedical implications of the co-evolution of pathogens and their hosts," Nature Genetics, vol. 32, no. 4, pp. 569-577, 2002.

[10] A. Casadevall and L.-A. Pirofski, "Host-pathogen interactions: basic concepts of microbial commensalism, colonization, infection, and disease," Infection and Immunity, vol. 68, no. 12, pp. 6511-6518, 2000.

[11] T. C. Schelling, The Strategy of Conflict, Harvard University Press, Cambridge, Mass, USA, 1960.

[12] J. Mehta, C. Starmer, and R. Sugden, "Focal points in pure coordination games: an experimental investigation," Theory and Decision, vol. 36, no. 2, pp. 163-185, 1994.

[13] R. E. Ley, D. A. Peterson, and J. I. Gordon, "Ecological and evolutionary forces shaping microbial diversity in the human intestine," Cell, vol. 124, no. 4, pp. 837-848, 2006.

[14] S. Falkow, "Is persistent bacterial infection good for your health?” Cell, vol. 124, no. 4, pp. 699-702, 2006.

[15] D. Kuklinska and M. Kilian, "Relative proportions of Haemophilus species in the throat of healthy children and adults," European Journal of Clinical Microbiology \& Infectious Diseases, vol. 3, no. 3, pp. 249-252, 1984.

[16] K. Binmore and L. Samuelson, "The evolution of focal points," Games and Economic Behavior, vol. 55, no. 1, pp. 21-42, 2006.

[17] R. Sugden and I. E. Zamarrón, "Finding the key: the riddle of focal points," Journal of Economic Psychology, vol. 27, no. 5, pp. 609-621, 2006.

[18] R. A. Welch, V. Burland, G. Plunkett III, et al., "Extensive mosaic structure revealed by the complete genome sequence of uropathogenic Escherichia coli," Proceedings of the National Academy of Sciences of the United States of America, vol. 99, no. 26, pp. 17020-17024, 2002.

[19] G. Pósfai, G. Plunkett III, T. Fehér, et al., "Emergent properties of reduced-genome Escherichia coli," Science, vol. 312, no. 5776, pp. 1044-1046, 2006.

[20] H. Brüssow, "Phage therapy: the Escherichia coli experience," Microbiology, vol. 151, no. 7, pp. 2133-2140, 2005.

[21] M. Ohnishi, K. Kurokawa, and T. Hayashi, "Diversification of Escherichia coli genomes: are bacteriophages the major contributors?" Trends in Microbiology, vol. 9, no. 10, pp. 481-485, 2001.

[22] D. A. Relman, "New technologies, human-microbe interactions, and the search for previously unrecognized pathogens," Journal of Infectious Diseases, vol. 186, supplement 2, pp. S254S258, 2002.

[23] J. Hacker and E. Carniel, "Ecological fitness, genomic islands and bacterial pathogenicity. A Darwinian view of the evolution of microbes," EMBO Reports, vol. 2, no. 5, pp. 376-381, 2001.

[24] P. J. Sansonetti, "War and peace at mucosal surfaces," Nature Reviews Immunology, vol. 4, no. 12, pp. 953-964, 2004.

[25] E. Rosenberg, O. Koren, L. Reshef, R. Efrony, and I. ZilberRosenberg, "The role of microorganisms in coral health, disease and evolution," Nature Reviews Microbiology, vol. 5, no. 5, pp. 355-362, 2007.

[26] M. E. Bianchi, "DAMPs, PAMPs and alarmins: all we need to know about danger," Journal of Leukocyte Biology, vol. 81, no. 1, pp. 1-5, 2007. 
[27] S.-H. Huang, "Infectomics-based new theory for dissecting the pathogenesis, prevention and therapeutics of microbial infections," in Teda-Watson International forum on Biotechnology and Biomedicine, vol. 4, pp. 73-74, Tianjin, China, 2005.

[28] S.-H. Huang, Y.-H. Chen, G. Kong, et al., "A novel genetic island of meningitic Escherichia coli $\mathrm{K} 1$ containing the ibeA invasion gene (GimA): functional annotation and carbonsource-regulated invasion of human brain microvascular endothelial cells," Functional \& Integrative Genomics, vol. 1, no. 5, pp. 312-322, 2001.

[29] J. Hejnova, U. Dobrindt, R. Nemcova, et al., "Characterization of the flexible genome complement of the commensal Escherichia coli strain A0 34/86 (O83:K24:H31)," Microbiology, vol. 151, no. 2, pp. 385-398, 2005.

[30] S. A. Joyce and D. J. Clarke, "A hexA homologue from Photorhabdus regulates pathogenicity, symbiosis and phenotypic variation," Molecular Microbiology, vol. 47, no. 5, pp. 1445$1457,2003$.

[31] G. Silvestri, "Naturally SIV-infected sooty mangabeys: are we closer to understanding why they do not develop AIDS?" Journal of Medical Primatology, vol. 34, no. 5-6, pp. 243-252, 2005.

[32] B. A. Webb, M. R. Strand, S. E. Dickey, et al., "Polydnavirus genomes reflect their dual roles as mutualists and pathogens," Virology, vol. 347, no. 1, pp. 160-174, 2006.

[33] K. S. Kobayashi and R. A. Flavell, "Shielding the double-edged sword: negative regulation of the innate immune system," Journal of Leukocyte Biology, vol. 75, no. 3, pp. 428-433, 2004.

[34] J. Demengeot, S. Zelenay, M. F. Moraes-Fontes, Í. Caramalho, and A. Coutinho, "Regulatory T cells in microbial infection," Springer Seminars in Immunopathology, vol. 28, no. 1, pp. 4150, 2006.

[35] R. Lucattini, V. A. Likić, and T. Lithgow, "Bacterial proteins predisposed for targeting to mitochondria," Molecular Biology and Evolution, vol. 21, no. 4, pp. 652-658, 2004.

[36] T. Uchiumi, T. Ohwada, M. Itakura, et al., "Expression islands clustered on the symbiosis island of the Mesorhizobium loti genome," Journal of Bacteriology, vol. 186, no. 8, pp. 24392448, 2004.

[37] M. C. W. Janssen, "Rationalizing focal points," Theory and Decision, vol. 50, no. 2, pp. 119-148, 2001.

[38] P. T. Leeson, C. J. Coyne, and P. J. Boettke, "Converting social conflict: focal points and the evolution of cooperation," The Review of Austrian Economics, vol. 19, no. 2-3, pp. 137-147, 2006.

[39] L. C. Gao, Principles of Systemics, Science Press, Beijing, China, 2005.

[40] A. M. Colman, “Thomas C. Schelling's psychological decision theory: introduction to a special issue," Journal of Economic Psychology, vol. 27, no. 5, pp. 603-608, 2006.

[41] D. E. Yirrell, E. Wright, L. A. Shafer, et al., "Association between active $\mathrm{GB}$ virus- $\mathrm{C}$ (hepatitis $\mathrm{G}$ ) infection and HIV-1 disease in Uganda," International Journal of STD \& AIDS, vol. 18, no. 4, pp. 244-249, 2007.

[42] T. N. Baral, S. Magez, B. Stijlemans, et al., "Experimental therapy of African trypanosomiasis with a nanobody-conjugated human trypanolytic factor," Nature Medicine, vol. 12, no. 5, pp. 580-584, 2006. 

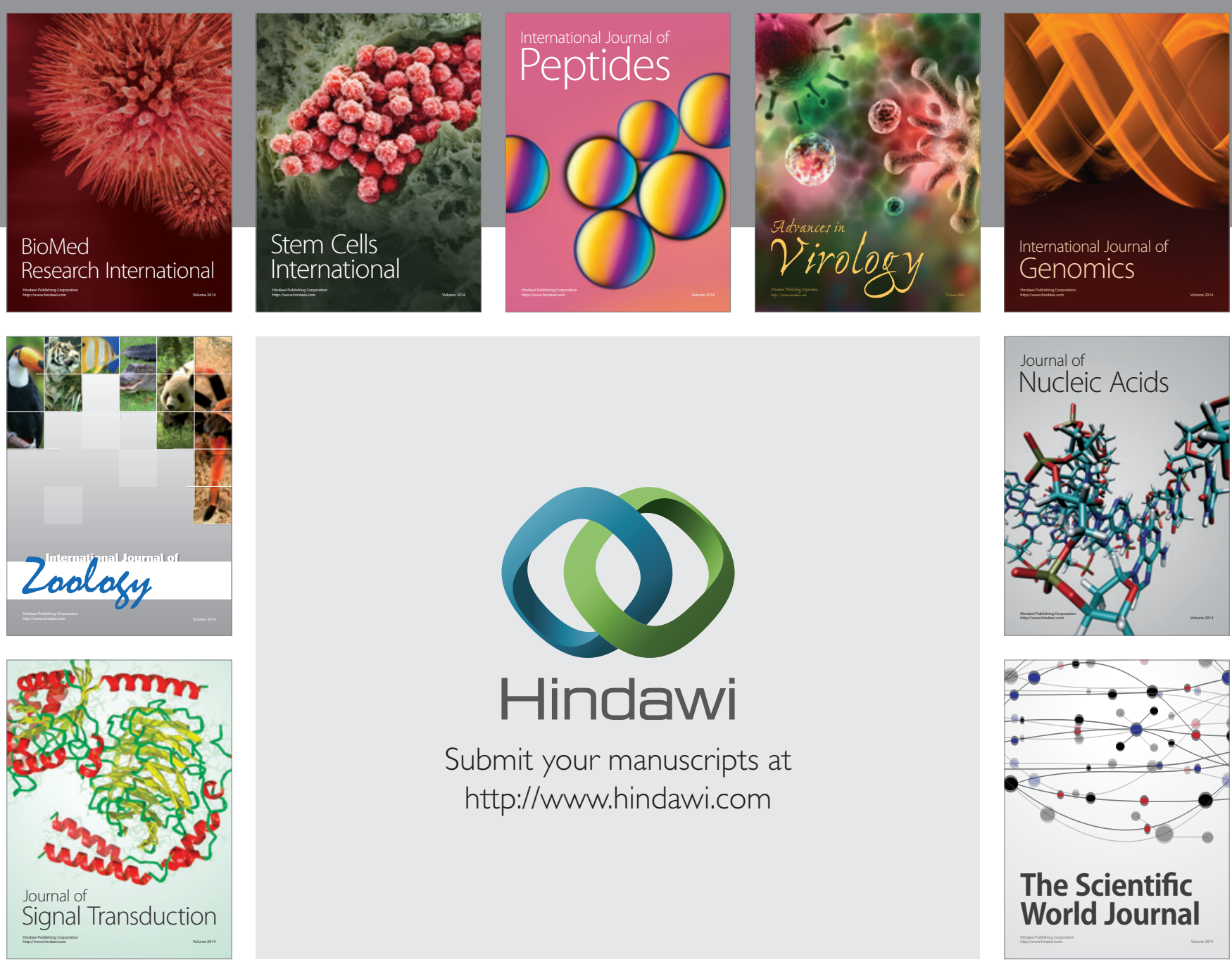

Submit your manuscripts at

http://www.hindawi.com
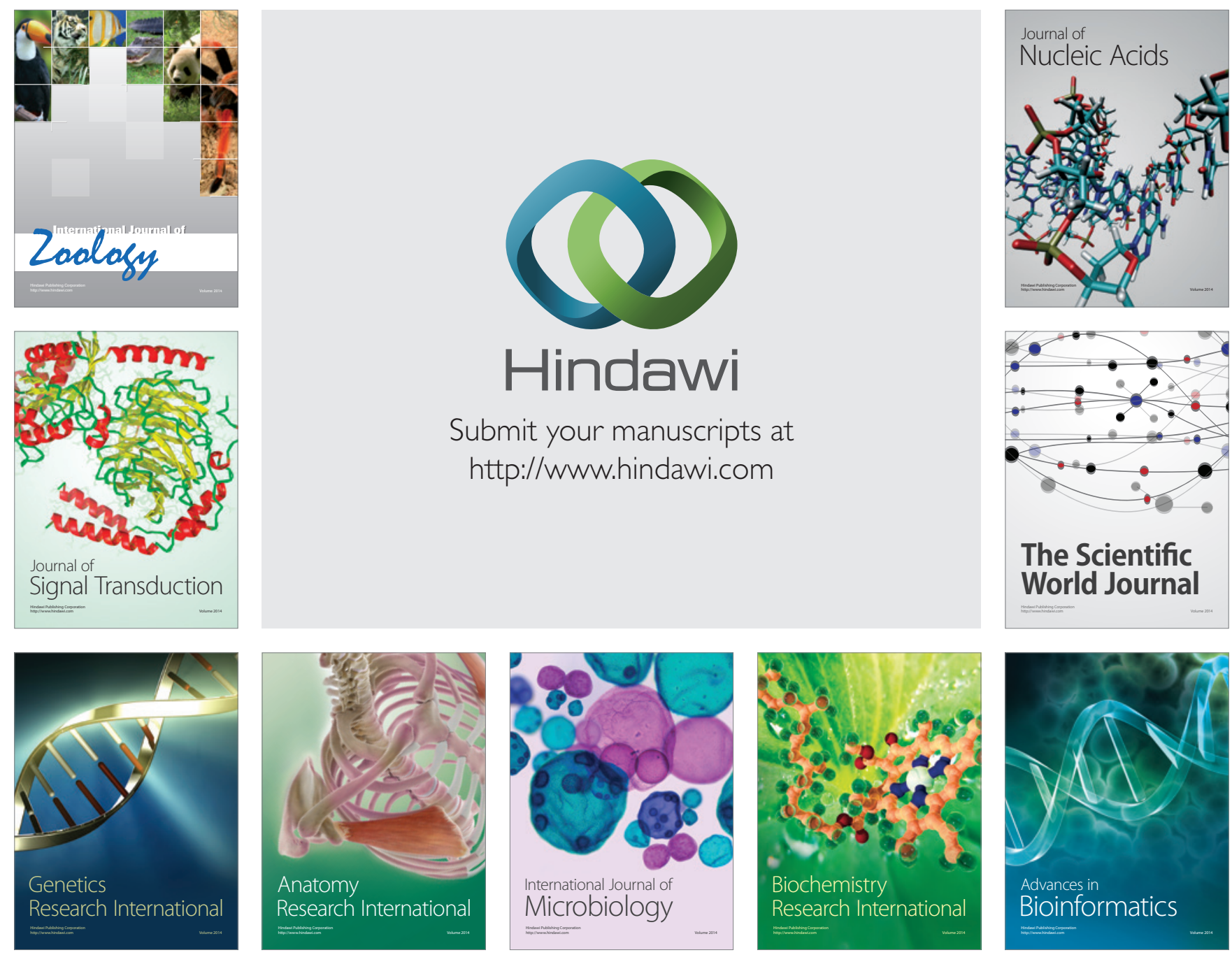

The Scientific World Journal
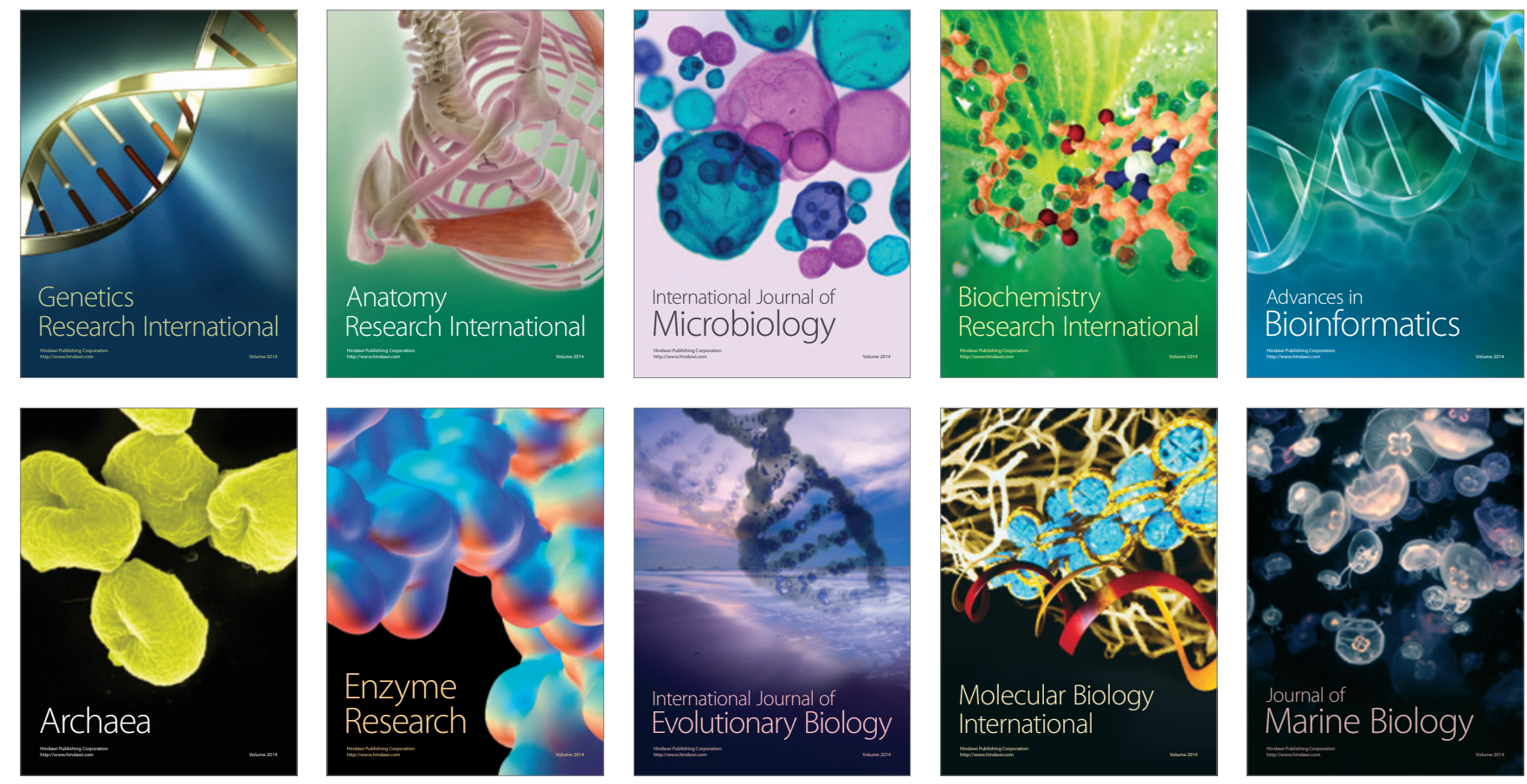